\title{
Multi-institutional analysis of bioimpedance spectroscopy in the early detection of breast cancer related lymphedema
}

\author{
Vicini $\mathrm{FA}^{1, *}$, Arthur $\mathrm{D}^{2}$, Shah $\mathrm{C}^{3}$, Anglin $\mathrm{BV}^{4}$, Curcio $\mathrm{LD}^{5}$, Laidley $\mathrm{AL}^{6}$, Beitsch $\mathrm{PD}^{7}$, Whitworth $\mathrm{P}^{8}$ and Lyden $\mathrm{M}^{9}$ \\ ${ }^{1}$ Michigan Healthcare Professionals/ 21st Century Oncology, Farmington Hills, Michigan \\ ${ }^{2}$ Department of Radiation Oncology, Virginia Commonwealth University, Virginia \\ ${ }^{3}$ Department of Radiation Oncology, Washington University School of Medicine, Saint Louis, Missouri \\ ${ }^{4}$ The Medical Center of Plano, Plano, Texas \\ ${ }^{5}$ Advanced Breast Care Specialists of Orange County, Mission Viejo, California \\ ${ }^{6}$ Texas Breast Specialists, Dallas, Texas \\ ${ }^{7}$ Dallas Surgical Group, Dallas, Texas \\ ${ }^{8}$ Nashville Breast Center, Nashville, Tennessee \\ ${ }^{9}$ Biostat Inc., Tampa, Florida
}

\begin{abstract}
Background: The purpose of this study was to evaluate bioelectrical impedance spectroscopy's (BIS) ability to detect and monitor extracellular fluid accumulation of the upper limb as it relates to the extent of loco-regional therapy. Methods: A total of 125 patients with breast cancer from 4 clinical practices were evaluated with BIS at baseline and following loco-regional procedures. In order to assess the ability of BIS to detect subclinical changes by treatment modality, the change in L-Dex score from baseline to measurements taken within 180 days following surgery were calculated. Results: Mean age was 55 years with 68 patients (54.4\%) undergoing sentinel lymph node (SLN) sampling while 57 (45.6\%) underwent an axillary dissection (ALND). Sixty-five patients (52\%) underwent radiation therapy (RT). Patients receiving RT had a significantly increased change in L-Dex score $(0.8 \mathrm{v} .-2.5, \mathrm{p}=0.03)$ compared with those patients not receiving RT. For all patients, ALND was associated with a significantly increased change in L-Dex score (5.0 v. 0.3, $\mathrm{p}=0.003$ ) compared with SLN. When stratifying by the number of nodes removed, a statistically significant increase in the change in L-Dex score was noted ( 0.4 v. 0.4 v. 4.3 v. $6.4, \mathrm{p}=0.04$ ) for $0-3,4-6,7-10$ and greater than 10 lymph nodes removed. Conclusions: In this limited analysis, L-Dex scores paralleled the extent of axillary sampling and the addition of radiation therapy; these results demonstrate that BIS can be used to monitor patients for the early onset of edema as differences emerged within 180 days of surgery.
\end{abstract}

Keywords: bioimpedance; breast cancer; lymphedema; early detection; subclinical assessment

\section{Introduction}

Over the past three decades, outcomes for women treated for breast cancer have continued to improve and with such improvements, an evolution in the assessment and management of treatment related toxicities has occurred [1]. One the most frequent and morbid complications associated with loco-regional therapy for breast cancer is lymphedema [2]. However, despite increasing concern regarding breast cancer-related lymphedema (BCRL), little has changed in the diagnostic assessment and treatment of the condition. Diagnostic modalities including arm circumference measurements, water displacement tanks and patient surveys have been utilized for several decades and have been employed to identify factors associated with the development of clinically significant BCRL [2, 3]. However, BCRL begins as a subclinical process due to an impairment of the lymphatic drainage system; this impairment causes an increase in the extracellular fluid which then leads to clinically detectable lymphedema and morbidities associated with BCRL such as pain, numbness and limb heaviness [4]. Once BCRL is clinically detectable, patients can develop chronic, irreversible BCRL which

*Corresponding author: Vicini FA, Michigan HealthCare Professionals/21st Century Oncology, 28595 Orchard Lake Road, Farmington Hills, MI 48331, Tel.: 248994 0632; Fax: 248553 7674; E-mail: fvicini@pol.net

Received 4 December 2012 Revised 20 December 2012 Accepted 5 February 2013 Published February 2013

Citation: Vicini FA, Arthur D, Shah C, Anglin BV, Curcio LD, Laidley AL, Beitsch PD, Whitworth P, Lyden M (2013) Multi-institutional analysis of bioimpedance spectroscopy in the early detection of breast cancer related lymphedema. J Cancer Res Ther 1: 1-7. doi:10.14312/20524994.2013-1

Copyright: (C) 2013 Vicini FA, et al. This is an open-access article distributed under the terms of the Creative Commons Attribution License, which permits unrestricted use, distribution and reproduction in any medium, provided the original author and source are credited. 
may include chronic skin changes, infection (cellulitis, lymphangitis), chronic pain and functional impairment of the affected limb [4]. It is therefore important to identify those patients at high risk for development of BCRL to help prevent the long term sequelae of BCRL.

One potential solution to reduce the incidence of clinically significant BCRL and the chronic morbidities associated is early detection and intervention. Randomized trials from La Comba et al. and Box et al. have identified reductions in chronic BCRL with early intervention with La Comba identifying an $18 \%$ reduction in BCRL at one year with early intervention and Box et al a 19\% reduction two years [5, 6]. Unfortunately, these trials had no method to evaluate patients with early signs of BCRL prior to enrollment and therefore the interventions did not risk stratify; this is likely secondary to traditional modalities being unable to identify subclinical BCRL. However, the findings from these trials are supported by a prospective analysis from Stout-Gergich et al. in which 196 women with early stage breast cancer underwent preoperative and postoperative volume assessments using perometry. Of these initial cases, 43 patients were identified with BCRL and treated with a 20-30 mmHg compression garment which reduced edema volumes without the need for further therapies [7]. At five years, $25 \%$ of patients had subclinical lymphedema ( $>3 \%$ volume change) and $5.6 \%$ developed advanced edema [8]; unfortunately, this study has limited ability to be extrapolated due to the expense associated with perometry and therefore its limited ability to be utilized in the clinic routinely.

Bioimpedance spectroscopy represents one of the newer diagnostic modalities for BCRL with the ability to detect subclinical extracellular fluid accumulation [9, $10,11]$. BIS uses a harmless low level electrical current to measure electrical impedance and therefore assess the extracellular volume. This is done by placing several electrodes on the body and measuring the voltage change (a measure of impedance) across the electrodes with a subsequent conversion of impedance to water volume based on conversion equations to create an L-Dex score. Using BIS would allow for the early detection of fluid accumulation by clinicians and subsequently, selective intervention in patients at high risk for the development of clinically significant BCRL.

At this time, limited clinical data is available utilizing BIS in the early detection of BCRL. A previous analysis from the Nashville Breast Center evaluated 64 patients with pre-treatment and post-treatment BIS assessments and assessed the feasibility ofimplementation of a BIS program. Changes in the L-Dex score, an index of fluid accumulation, acquired from BIS were associated with more aggressive regional therapy including ALND v. sentinel lymph node $(\mathrm{SLN})(\mathrm{p}=0.08)$ and increasing nodes sampled $(\mathrm{p}=0.09)$ but did not reach statistical significance, likely due to small sample size [12]. Based on these findings, our group has hypothesized that BIS has the sensitivity to detect changes in extracellular volume within 6 months of completion of loco-regional therapy and that L-Dex score changes are correlated with the aggressiveness of loco-regional therapy. Therefore, the purpose of the present study was to perform an exploratory analysis of data collected from multiple institutions in order to evaluate the BIS's ability to detect extracellular fluid accumulation of the upper limb and to determine if changes in L-Dex score related to the extent of loco-regional therapy, allowing for detection of a possible high risk cohort who may warrant more aggressive BCRL screening.

\section{Materials and methods}

Four centers that had experience with the L-Dex U400 (Impedimed, Brisbane, Australia) were chosen to participate in providing retrospective data from patients who received L-Dex procedures. This analysis represents a retrospective review of the first 125 patients who were prospectively seen according to standard of care. Approval for data collection and use for each clinical site was obtained via each site's institutional review board (IRB or institutional ethics committee). Inclusion criteria consisted of patients of at least 18 years of age who underwent breast cancer surgery with unilateral SLN biopsy or ALND and who had a pre-surgical L-Dex measurement as well as at least 2 post-surgical measurements. Patients were eligible if they received either a mastectomy or breast conservation therapy. Post-mastectomy reconstruction was allowed with either tissue expander/implant or autologous reconstruction; however, information on reconstruction technique utilized was not available. Systemic therapy including chemotherapy and hormonal therapy was allowed and could be delivered as neo-adjuvant or adjuvant therapy. Exclusion criteria included implantable electronic devices (i.e., pacemakers), bilateral disease, pregnancy, renal failure and heart failure. De-identified data was collected from a random selection of qualified patients at each center by an independent study coordinator. Each center received an IRB approval which did not require individual informed consent from each patient to use their deidentified information for the analysis.

Data collected on each patient included age, height, weight and menopausal status. Surgical parameters including the type and number of procedures and the number of nodes removed were recorded on each patient along with their pre-surgical (baseline) and all available post-surgical L-Dex measurements. A subset of patients with available records indicating whether systemic therapy was administered had the types and dates of administration recorded. Any available information on prescribed lymphedema interventions whether prophylactic or treatment of symptoms was abstracted, however this information was sparse and not consistently recorded in the medical record. 
All L-Dex measurements were obtained utilizing the same procedure described in Vicini et al. [12]. Patients were tested in a supine position on a non-metallic surface with feet shoulder width apart and hands by their sides. Electrodes were placed on the skin on the midline dorsal surface of the wrist at the level of the ulnar styloid process and on the skin on the midline anterior surface of the ankle at the level of the medial and lateral malleolus bones. Following electrode placement, alligator clips were attached to each electrode. Of note, the analysis by Vicini et al. utilized a single frequency bioelectrical device while current assessments were performed using the L-Dex U400 (Impedimed Ltd., Brisbane, Australia) which is a bioelectrical impedance spectroscopy device that provides L-Dex scores; the L-Dex score represents a linearly scaled form of the lymphedema index ratios (LIR), which for the purpose of comparison to previous studies can be used interchangeably [13]. The baseline L-Dex score, measuring impedance, can be a positive or negative score with an increase in extracellular fluid reflected in an overall increase in the L-Dex score; a change of 10 in L-Dex score has been utilized as a change indicative of BCRL.

\section{Statistical Analyses}

The analysis included all patients that met the inclusion criteria. Body mass index (BMI) was calculated based on patient height and weight. Patient and surgical characteristics were summarized descriptively for all subjects. Surgical characteristics were compared by surgical technique and administration of radiation therapy using Fisher's exact test for categorical variables and Wilcoxon rank sum for continuous variables. An analysis was performed comparing baseline L-Dex score to the first assessment within 180 days (Table 1 ) and also to the first post-surgical assessments regardless of the time from surgery (Table 2); for each analysis only the first post-treatment L-Dex measurement was utilized. A

Table 1 Changes in L-Dex score by treatment characteristic comparing baseline assessment to post-treatment assessment within 180 days of surgery

\begin{tabular}{|c|c|c|c|c|c|c|c|c|c|c|c|}
\hline & \multicolumn{3}{|c|}{ Baseline L-Dex Score } & \multicolumn{8}{|c|}{ Change in L-Dex Score } \\
\hline & $\mathrm{N}$ & $\begin{array}{l}\text { Mean/ } \\
\text { Median }\end{array}$ & Range & $\mathrm{N}$ & Mean & Median & Std & $\begin{array}{l}\text { Min } \\
\text { change }\end{array}$ & $\begin{array}{l}\text { Max } \\
\text { change }\end{array}$ & $\begin{array}{l}\text { Within } \\
\text { Group } \\
\text { p-value }\end{array}$ & $\begin{array}{l}\text { Between } \\
\text { Groups } \\
\text { p-value }\end{array}$ \\
\hline All patients & 125 & $0.4 /-0.4$ & $-13.7-28.3$ & 84 & 2.0 & 0.3 & 6.83 & -16.8 & 20.2 & 0.51 & \\
\hline \multicolumn{12}{|l|}{ Surgery } \\
\hline Lumpectomy & 51 & $0.1 /-0.5$ & $-10.5-14.8$ & 35 & 1.8 & 0.2 & 5.70 & -7.8 & 15.1 & 0.61 & 0.97 \\
\hline Mastectomy & 74 & $0.5 /-0.4$ & $-13.7-28.3$ & 49 & 2.2 & 0.9 & 7.60 & -16.8 & 20.2 & 0.77 & \\
\hline \multicolumn{12}{|l|}{ Axillary procedure } \\
\hline Sentinel node only & 68 & $0.6 /-0.5$ & $-10.5-28.3$ & 54 & 0.3 & -0.4 & 5.85 & -16.8 & 15.3 & 0.27 & $0.003^{*}$ \\
\hline Axillary excision & 57 & $0.1 /-0.4$ & $-13.7-14.8$ & 30 & 5.0 & 2.5 & 7.53 & -7.8 & 20.2 & $0.003^{*}$ & \\
\hline \multicolumn{12}{|l|}{ Number of nodes } \\
\hline $0-3$ & 51 & $0.2 /-0.5$ & $-10.5-11.4$ & 40 & 0.4 & -0.3 & 4.85 & -7.6 & 15.1 & 0.52 & $0.04^{*}$ \\
\hline $4-6$ & 23 & $1.2 /-0.8$ & $-6.2-28.3$ & 18 & 0.4 & -0.5 & 6.80 & -16.8 & 15.2 & 1.0 & \\
\hline $7-10$ & 17 & $0.3 /-0.3$ & $-10.5-14.8$ & 11 & 4.3 & 2.3 & 8.15 & -6.3 & 19.6 & 0.34 & \\
\hline$>10$ & 34 & $0.0 /-0.5$ & $-13.7-10.4$ & 15 & 6.4 & 5.9 & 8.50 & -7.8 & 20.2 & $0.04^{*}$ & \\
\hline \multicolumn{12}{|c|}{ Pairwise comparison of number of nodes removed } \\
\hline $\begin{array}{l}\text { Number of nodes } \\
\text { removed }\end{array}$ & & $0-3$ & $4-6$ & $7-10$ & $>10$ & & & & & & \\
\hline $0-3$ & & -- & 0.82 & 0.13 & $0.008^{*}$ & & & & & & \\
\hline $4-6$ & & -- & -- & 0.28 & $0.04^{*}$ & & & & & & \\
\hline $7-10$ & & -- & -- & -- & 0.48 & & & & & & \\
\hline
\end{tabular}

Abbreviations: $\mathrm{Std}=$ standard deviation, $\mathrm{N}=$ number of patients

$*=$ statistically significant 
Table 2 Changes in L-Dex score by treatment characteristic comparing baseline assessment to first post-treatment assessment

\begin{tabular}{|c|c|c|c|c|c|c|c|c|c|c|c|}
\hline & Base & e L-Dex Scor & & Chan & $L-D e x s c o$ & & & & & & \\
\hline Parameter & $N$ & $\begin{array}{l}\text { Mean/ } \\
\text { median }\end{array}$ & Range & $N$ & Mean & Median & Std & $\begin{array}{l}\text { Min } \\
\text { change }\end{array}$ & $\begin{array}{l}\text { Max } \\
\text { change }\end{array}$ & $\begin{array}{l}\text { Within } \\
\text { group } \\
\text { p-value }\end{array}$ & $\begin{array}{l}\text { Between } \\
\text { groups } \\
\text { p-value }\end{array}$ \\
\hline All patients & 125 & $0.4 /-0.4$ & $\begin{array}{l}-13.7- \\
28.3\end{array}$ & 125 & 2.0 & 0.5 & 6.22 & -16.8 & 20.2 & 0.20 & \\
\hline \multicolumn{12}{|l|}{ Surgery } \\
\hline Lumpectomy & 51 & $0.1 /-0.5$ & $\begin{array}{l}-10.5- \\
14.8\end{array}$ & 51 & 1.3 & 0.0 & 5.43 & -11.3 & 15.1 & 1.0 & 0.37 \\
\hline Mastectomy & 74 & $0.5 /-0.4$ & $\begin{array}{l}-13.7- \\
28.3\end{array}$ & 74 & 2.5 & 2.1 & 6.71 & -16.8 & 20.2 & 0.12 & \\
\hline \multicolumn{12}{|l|}{ Axillary procedure } \\
\hline Sentinel node only & 68 & $0.6 /-0.5$ & $\begin{array}{l}-10.5- \\
28.3\end{array}$ & 68 & 0.3 & -0.4 & 5.30 & -16.8 & 15.3 & 0.14 & $0.0002^{*}$ \\
\hline Axillary excision & 57 & $0.1 /-0.4$ & $\begin{array}{l}-13.7- \\
14.8\end{array}$ & 57 & 4.0 & 2.7 & 6.66 & -11.3 & 20.2 & $0.0002^{*}$ & \\
\hline \multicolumn{12}{|l|}{ Number of nodes } \\
\hline $0-3$ & 51 & $0.2 /-0.5$ & $\begin{array}{l}-10.5- \\
11.4\end{array}$ & 51 & 0.5 & -0.3 & 4.58 & -7.6 & 15.1 & 0.39 & $0.006^{*}$ \\
\hline $4-6$ & 23 & $1.2 /-0.8$ & $-6.2-28.3$ & 23 & 0.3 & -0.8 & 6.07 & -16.8 & 15.2 & 0.83 & \\
\hline $7-10$ & 17 & $0.3 /-0.3$ & $\begin{array}{l}-10.5- \\
14.8\end{array}$ & 17 & 2.9 & 2.3 & 7.58 & -11.3 & 19.6 & 0.21 & \\
\hline$>10$ & 34 & $0.0 /-0.5$ & $\begin{array}{l}-13.7- \\
10.4\end{array}$ & 34 & 4.9 & 4.4 & 6.85 & -7.8 & 20.2 & $0.003^{*}$ & \\
\hline \multicolumn{12}{|c|}{ Pairwise comparison of number of nodes removed } \\
\hline $\begin{array}{l}\text { Number of nodes } \\
\text { removed }\end{array}$ & & $0-3$ & $4-6$ & $7-10$ & $>10$ & & & & & & \\
\hline $0-3$ & & -- & 0.99 & 0.12 & $0.001^{*}$ & & & & & & \\
\hline $4-6$ & & -- & -- & 0.22 & $0.01^{*}$ & & & & & & \\
\hline $7-10$ & & -- & -- & -- & 0.31 & & & & & & \\
\hline
\end{tabular}

Abbreviations: Std= standard deviation, $\mathrm{N}=$ number of patients

*= statistically significant

180 day time point was selected to determine if changes in L-Dex scores emerged within 6 months of surgery in order to evaluate the BIS's ability to detect early subclinical changes. Changes in L-Dex score within surgical treatment categories and between them were analyzed using nonparametric tests (Wilcoxon Signed Rank tests for within surgical categories and Wilcoxon Rank Sum (2 levels) or Kruskall-Wallis (> 2 levels) tests for between categories). Changes from pre-radiation to the last available L-Dex for patients who received radiation and changes from the first post-surgical to last available L-Dex for those patients who did not receive radiation were calculated. These changes were determine to the effect due to radiation via an analysis of covariance where baseline L-Dex score and number of nodes surgically removed were included as covariates in the model. The patient selection was done according to an independent random rule and data abstraction was done by independent data monitors.
Impedimed supported financially the time spent to collect and analyze data, but did not influence the subjects who were selected or data that was abstracted.

\section{Results}

Patient characteristics are presented in Table 3. A total of 125 patients was evaluated with a mean/median age of $55 / 55.3$ years old. The mean body mass index was 27.8 $\mathrm{kg} / \mathrm{m} 2$ with $30.4 \%$ of patients having a BMI between 25 and 30 and $26.4 \%$ of patients having a BMI of 30 or greater. Fifty-one patients (40.8\%) underwent lumpectomy and $74(59.2 \%)$ mastectomy with 37 patients $(29.6 \%)$ requiring more than one procedure; of these 37 patients, 10 underwent re-excision. Sixty-eight patients (54.4\%) underwent sentinel lymph node (SLN) sampling while 57 (45.6\%) underwent an axillary lymph node dissection (ALND). The mean number of nodes sampled was 7.9 (range:1.0-37.0) with 40.8\%,18.4\%, 13.6\% and 27.2\% 
of patients having $0-3,4-6,7-9$ or greater than 10 lymph nodes sampled.

Patient characteristics by surgical intervention are presented in Table 4. Lumpectomy patients were more likely to have undergone re-excision $(15.7 \%$ v. $2.7 \%$,

Table 3 Patient characteristics

\begin{tabular}{ll}
\hline Age (years) & \\
\hline Mean +/-SD & $55.0+/-12.0$ \\
Median & 55.3 \\
Range & $29.9-86.8$ \\
$<40$ years & $10(8.0 \%)$ \\
$40-<50$ years & $40(32.0 \%)$ \\
$50-<60$ years & $28(22.4 \%)$ \\
$60-<70$ years & $32(25.6 \%)$ \\
$>=70$ years & $14(11.2 \%)$ \\
$<50$ years & $50(40.0 \%)$ \\
$>=50$ years & $74(59.2 \%)$ \\
Height $(\mathrm{cm})$ & \\
Mean $+/-$ sd & \\
Median & $164.1+/-7.1$ \\
Weight $(\mathrm{kg})$ & 164.1 \\
Mean $+/-\mathrm{SD}$ & \\
Median & \\
Range & \\
BMI $(\mathrm{kg} / \mathrm{m} 2)$ & $74.5+/-17.3$ \\
Mean +/-SD & 71.2 \\
Median & $47.6-142.9$ \\
Range & \\
$<25$ & \\
$25-<30$ & $27.8+/-6.9$ \\
& 26.5 \\
\hline & $17.7-52.4$ \\
& $48(38.4 \%)$ \\
& $38(30.4 \%)$ \\
& \\
& \\
& \\
&
\end{tabular}

$\mathrm{p}=0.02)$, have sentinel lymph node assessment $(66.7 \%$ v. $45.9 \%, \mathrm{p}=0.03$ ) and fewer nodes sampled (5.5 v. 9.5, $\mathrm{p}=0.0008)$. Of note, lumpectomy patients were more likely to have undergone radiation therapy $(97.7 \%$ v. $42.3 \%$, $\mathrm{p}<0.001)$. Sixty-five patients $(52 \%)$ underwent radiation therapy (RT) with RT patients being more likely to have undergone lumpectomy $(66.2 \%$ v. $3.2 \%, \mathrm{p}<0.001)$ and axillary dissection $(41.5 \%$ v. $19.4 \%, \mathrm{p}=0.04)$ compared with patients not receiving RT (Table 4). However, despite RT patients more frequently undergoing ALND, no difference in the mean number of nodes sampled (7.7 v. $5.4, p=0.14$ ) was noted compared with patients not receiving $\mathrm{RT}$.

When controlling for baseline L-Dex score and the number of nodes removed, patients receiving RT had a significantly increased change (pre v. post radiation treatment) in mean L-Dex score $(-2.5 \mathrm{v}$. $0.8, \mathrm{p}=0.03)$ compared with those patients not receiving RT. Changes in mean L-Dex score stratified by treatment characteristics are presented in Tables 1 and 2 . Table 1 presents changes in mean L-Dex score while comparing baseline BIS assessment and the first assessment within 180 days of surgery. In all patients, no difference in the change in the score was noted by surgical procedure (lumpectomy $1.8 \mathrm{~V}$. mastectomy 2.2, $\mathrm{p}=0.97)$; however, ALND was associated with a significantly increased change in mean L-Dex score $(0.3$ v. $5.0, p=0.003)$ compared with SLN biopsy. When stratifying by the number of nodes removed, a statistically significant increase in the change in mean L-Dex score was noted ( 0.4 v. 0.4 v. 4.3 v. $6.4, p=0.04$ ) for 0-3, 4-6, 7-10 and greater than 10 lymph nodes removed; pairwise comparison found significant differences for 0-3 $(p=0.008)$ and 4-6 $(p=0.04)$ nodes removed compared with greater than 10 nodes removed. Similar findings were noted when evaluating the impact of ALND $(0.3 \mathrm{v}$. $4.0, p=0.0002)$ and nodes removed ( 0.5 v. 0.3 v. 2.9 v. 4.9, $\mathrm{p}=0.006$ ) when comparing the baseline BIS assessment to the first post-surgical assessment regardless of when the assessment was performed post-operatively (Table 2); pairwise comparison found significant differences for 0-3 $(p=0.001)$ and 4-6 $(p=0.01)$ nodes removed compared with greater than 10 nodes removed.

\section{Discussion}

The results of this exploratory analysis demonstrate several key points; the first finding is that BIS can detect changes in L-Dex score within 6 months of surgery, well before clinical changes develop in patients. This change in L-Dex score is associated with an increased amount of extracellular fluid, a finding seen with subclinical BCRL, suggesting that L-Dex values do change following treatment in conjunction with increasing arm volumes. These findings are consistent with previously reported series including one from Cornish et al; in this analysis, 102 patients were evaluated by the study concluding that multi-frequency bioimpedance was able to detect BCRL 10 months prior to clinically apparent symptoms or signs [14]. It should be noted that the changes in L-Dex score were less than the threshold of 10 (which has not been consistently validated) and further studies are required to determine that changes less than 10 in L-Dex score have a similar relationship to BCRL as the cutoff of 10 .

A second major conclusion from this analysis is that that BIS can identify differences in L-Dex score based on the aggressiveness of loco-regional therapy, with patients undergoing ALND and having more lymph nodes removed, having statistically significant increases in L-Dex score within 6 months of surgery. Further, radiation therapy was associated with an increased change in L-Dex score (0.8 v.-2.5, p=0.03) compared with those patients not receiving radiation therapy. These findings are consistent with large randomized series that have confirmed more extensive axillary surgery and radiation therapy are associated with higher rates of BCRL and Vicini et al. which found non-significant associations for increased L-Dex scores with more aggressive axillary surgery using BIS $[12,15,16]$. Based on our analysis and those previously mentioned, patients undergoing more 
Table 4 Patient characteristics by surgical intervention and delivery of radiation therapy

\begin{tabular}{|c|c|c|c|c|c|c|}
\hline Number of Procedures & Lumpectomy & Mastectomy & $p$-value & $\begin{array}{l}\text { Radiation } \\
\text { Therapy }\end{array}$ & $\begin{array}{l}\text { No Radiation } \\
\text { Therapy }\end{array}$ & $p$-value \\
\hline 1 Procedure & $40(78.4 \%)$ & $48(64.9 \%)$ & 0.11 & $45(69.2 \%)$ & $15(48.4 \%)$ & 0.07 \\
\hline$>1$ Procedure & $11(21.6 \%)$ & $26(35.1 \%)$ & & $20(30.8 \%)$ & $16(51.6 \%)$ & \\
\hline Re-excision & $8(15.7 \%)$ & $2(2.7 \%)$ & $0.02^{*}$ & $8(12.3 \%)$ & $2(6.5 \%)$ & 0.49 \\
\hline \multicolumn{7}{|l|}{ Axillary procedure } \\
\hline Sentinel node & $34(66.7 \%)$ & $34(45.9 \%)$ & $0.03^{*}$ & $38(58.5 \%)$ & $25(80.6 \%)$ & $0.04^{*}$ \\
\hline Axillary excision & $17(33.3 \%)$ & $40(54.1 \%)$ & & $27(41.5 \%)$ & $6(19.4 \%)$ & \\
\hline Number of nodes & & & $0.0008^{*}$ & & & 0.14 \\
\hline Mean & 5.5 & 9.5 & & 7.7 & 5.4 & \\
\hline Median & 3 & 7.5 & & 4 & 3 & \\
\hline Range & $1-32$ & $1-37$ & & $1-37$ & $1-27$ & \\
\hline $0-3$ & $29(56.9 \%)$ & $22(29.7 \%)$ & $0.008^{*}$ & $28(43.1 \%)$ & $17(54.8 \%)$ & 0.19 \\
\hline $4-6$ & $10(19.6 \%)$ & $13(17.6 \%)$ & & $11(16.9 \%)$ & $8(25.8 \%)$ & \\
\hline $7-10$ & $4(7.8 \%)$ & $13(17.6 \%)$ & & $10(15.4 \%)$ & $1(3.2 \%)$ & \\
\hline$>=10$ & $8(15.7 \%)$ & $26(35.1 \%)$ & & $16(24.6 \%)$ & $5(16.1 \%)$ & \\
\hline \multicolumn{7}{|l|}{ Surgery } \\
\hline Lumpectomy & -- & -- & & $43(66.2 \%)$ & $1(3.2 \%)$ & $<0.0001^{*}$ \\
\hline Mastectomy & -- & -- & & $22(33.8 \%)$ & $30(96.8 \%)$ & \\
\hline \multicolumn{7}{|l|}{ Radiation } \\
\hline Yes & $43(97.7 \%)$ & $22(42.3 \%)$ & $<.0001$ & -- & -- & \\
\hline No & $1(2.3 \%)$ & 30 (57.7\%) & & -- & -- & \\
\hline
\end{tabular}

*- Statistically significant

aggressive axillary surgery (ALND, > $10 \mathrm{LN}$ sampled) and radiation therapy are at higher risk for developing BCRL and increases in L-Dex score [15]; placing such patients into a screening program would allow for early detection of BCRL, allowing for early management and potential prevention of chronic BCRL [8]. Before developing such screening programs, it has to be demonstrated, however that it is feasible to build such a program. This analysis, by its multi-institutional design and heterogeneity in clinical settings, confirms that BIS can be implemented easily in a variety of clinics as previously noted by Vicini et al. [12]. Unlike other techniques with high sensitivity (such as perometry), BIS can be easily implemented into the clinic with limited space requirements for setting up, low initial costs and relatively small increases in workflow (5-10 $\min )[12]$.

While the important finding of this analysis was the ability of BIS to detect subclinical BCRL within 6 months of surgery, the question arises as to the importance of this observation and whether early detection and therefore, early intervention provides a benefit to breast cancer survivors. As previously mentioned, a randomized trial from Lacomba et al. evaluated 120 women undergoing ALND and randomized patients to a program of manual lymphatic drainage, massage and exercise along with BCRL education following surgery or BCRL education only. At one year, $25 \%$ of patients in the control group (education only) developed BCRL compared with $7 \%$ in the intervention arm (HR 0.28, p=0.01) [6]. A second randomized trial of 65 women treated with ALND compared prospective physiotherapy with the surveillance; with two year follow-up, the incidence of chronic BCRL was $11 \%$ with physiotherapy compared with $30 \%$ with no early intervention [7]. Also, a large retrospective analysis of 1,713 patients with early stage breast cancer found that patients presenting with low volume BCRL had an $80 \%$ freedom from progression to more advanced BCRL at one year and $67 \%$ at five years [17]. These finding further suggest that the BIS could be used as part of a BCRL screening program for patients at high risk (ALND, > 10 nodes sampled, radiation 
therapy) to develop BCRL; patients identified with BCRL in the early phase of the disease process would be able to undergo early intervention potentially limiting the chronic sequelae of the disease.

There are limitations to this analysis. While the data were collected prospectively and entered into patient medical records as part of the standard of care, the data for this analysis represents an unplanned retrospective chart review and is subject to the limitations and biases of such an analysis. Further, although our series represents the largest in the literature to date, additional, larger series are required to confirm the findings of this analysis. Further, due to the limited follow-up, long term data with regards to the development of clinical BCRL are unavailable. Finally, although radiation therapy was evaluated as a factor associated with changes in L-Dex, detailed analysis of the delivery of radiation therapy was not available. Despite these limitations, this study represents the largest clinical study to date assessing the ability of BIS to detect early extracellular fluid changes. Future randomized prospective trials should be developed in order to examine the use of BIS in the detection of subclinical BCRL and further, the impact of early intervention in patients identified using BIS compared with intervention at the time of clinical diagnosis of BCRL.

\section{Conclusions}

In this multi-institutional analysis, L-Dex scores generated from multi-frequency bioimpedance spectroscopy mirrored the aggressiveness of loco-regional therapy with respect to the type and extent of nodal sampling and radiation therapy. These findings suggest that BIS can be used in the early detection of BCRL and should be implemented before and following loco-regional therapies. Further studies are needed to help validate the extent, degree and chronologic time frame of these changes to help define recommendations for closer monitoring and possible early intervention and to compare them to concurrent clinical assessments.

\section{Financial support}

Analysis supported by Impedimed (Brisbane, Australia)

\section{Conflicts of interest}

The authors wish to express that they have no conflict of interest.

\section{References}

[1] Ho A, Morrow M (2011) The evolution of the locoregional therapy of breast cancer. Oncologist 16:1367-1379.

[2] Lawenda BD, Mondry TE, Johnstone PA (2009) Lymphedema: a primer on the identification and management of a chronic condition in oncologic treatment. CA Cancer J Clin 59:8-24.

[3] Gärtner R, Jensen MB, Kronborg L, Ewertz M, Kehlet H, et al. (2010) Self-reported arm-lymphedema and functional impairment after breast cancer treatment--a nationwide study of prevalence and associated factors. Breast 19:506-515.
[4] McLaughlin SA, Cohen S, Van Zee KJ. Lymphedema (2010) In Diseases of the Breast (4th edn) HarrisJr, Lippincott Williams and Wilkins, Philadelphia, PA.

[5] Torres Lacomba M, Yuste Sánchez MJ, Zapico Goñi A, Prieto Merino D, Mayoral del Moral O, et al. (2010) Effectiveness of early physiotherapy to prevent lymphoedema after surgery for breast cancer: randomised, single blinded, clinical trial. BMJ 340:b5396.

[6] Box RC, Reul-Hirche HM, Bullock-Saxton JE, Furnival CM (2002) Physiotherapy after breast cancer surgery: results of a randomised controlled study to minimise lymphoedema. Breast Cancer Res Treat 75:51-64.

[7] Stout Gergich NL, Pfalzer LA, McGarvey C, Springer B, Gerber LH, et al. (2008) Preoperative assessment enables the early diagnosis and successful treatment of lymphedema. Cancer 112:2809-2819.

[8] Stout NL, Pfalzer L, Levy E, et al. (2011) Five-year preliminary outcomes of a prospective surveillance model to reduce upper extremity morbidity related to breast cancer treatment. San Antonio Breast Cancer Symposium. San Antonio, Texas, Abstract P4-12-08.

[9] Ward LC (2012) Early diagnosis in latent phase. In Lee BB, Bergan J, Rockson SG, eds. Lymphedema: A concise compendium of theory and practice. (1st edn). London, UK.

[10] Cornish BH, Chapman M, Hirst C, Mirolo B, Bunce IH, et al. (2001) Early diagnosis of lymphedema using multiple frequency bioimpedance. Lymphology 34:2-11.

[11] Ward LC, Dylke E, Czerniec S, Isenring E, Kilbreath SL, et al. (2011) Confirmation of the reference impedance ratios used for assessment of breast cancer-related lymphedema by bioelectrical impedance spectroscopy. Lymphat Res Biol 9:47-51.

[12] Vicini F, Shah C, Lyden M, Whitworth P (2012) Bioelectrical impedance for detecting and monitoring patients for the development of upper limb lymphedema in the clinic. Clin Breast Cancer 12:133-137.

[13] Gaw R, Box R, Cornish B (2011) Bioimpedance in the assessment of unilateral lymphedema of a limb: the optimal frequency. Lymphat Res Biol 9:93-99.

[14] Cornish BH, Chapman M, Hirst C, Mirolo B, Bunce IH, et al. (2001) Early diagnosis of lymphedema using multiple frequency bioimpedance. Lymphology 34:2-11.

[15] Ashikaga T, Krag DN, Land SR, Julian TB, Anderson SJ, et al. (2010) Morbidity results from the NSABP B-32 trial comparing sentinel lymph node dissection versus axillary dissection. J Surg Oncol 102:111-118.

[16] Coen JJ, Taghian AG, Kachnic LA, Assaad SI, Powell SN (2003) Risk of lymphedema after regional nodal irradiation with breast conservation therapy. Int Int J Radiat Oncol Biol Phys 55: 12091215.

[17] Bar Ad V, Cheville A, Solin LJ, Dutta P, Both S, et al. (2010) Time course of mild arm lymphedema after breast conservation treatment for early-stage breast cancer. Int J Radiat Oncol Biol Phys 76:85-90. 